\title{
A Comparison of Optical Light Transmission and Dynamic Viscoelastic Properties for Determining Transitions in Thermotropic Liquid Crystalline Polymers
}

\author{
Paul Driscoll, Toshiro Masuda, Akira Furukawa,* \\ Robert W. Lenz, ${ }^{* *}$ and Subrata BHATTACHARYA** \\ Research Center for Medical Polymers and Biomaterials, Kyoto University, \\ Shogoin, Sakyo-ku, Kyoto 606, Japan \\ *Mitsubishi Paper Mills, Ltd., Tsukuba Lab., \\ Wadai 4-10-6, Tsukuba-shi, Ibaraki, 300-42, Japan \\ **Polymer Science and Engineering, University of Massachusetts, \\ Amherst, MA 01003, U.S.A.
}

(Received November 22, 1989)

\begin{abstract}
Optical light transmission and dynamic viscoelastic properties are compared over the range of flow temperatures for two homogeneous thermotropic liquid crystalline polymers, TLCP, of identical structure but of different molecular weight. In the previous work involving only the higher molecular weight polymer, a Rheometerics Mechanical Spectrometer was used to study the dynamic viscoelastic properties, and this method proved to be a useful means for characterizing the isotropization process for this type of polymer. In the current work, parallel polarized light transmission was studied as another useful means of following the transition processes in homogeneous TLCP's. The light transmission data so obtained are compared with the light transmission data using crossed polarizers reported by previous workers for LCPs. Also included in this study was the characterzation of the LC transitions by DSC and polarized light microscopy.

KEY WORDS Thermotropic Liquid Crystalline Polymer / Dynamic Viscoelasticity / Optical Light Transmission / Effect of Temperature on Phase Separation / Molecular Weight Effects /
\end{abstract}

In the previous paper ${ }^{1}$ we described the intermediate temperature minima of the dynamic viscoelastic properties of poly[( $p$ fluorophenylene sulfonyl)- $p$-phenylene-1,10decamethylene-bis(4-oxybenzoate)], PFSPDB, of relatively high molecular weight of the following structure:

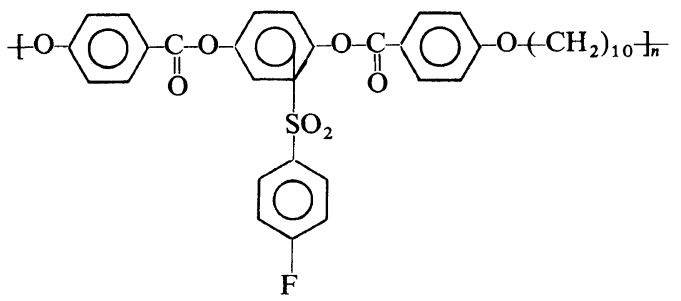

The sample described in that paper will be referred to as Polymer I in this study.
In was concluded that the minima observed, and the corresponding maxima, were caused by morphological changes of the polymer with temperature, more specifically by the changes associated with the liquid crystalline (LC) properties of this polymer. Although polarized light microscopy showed little texture change with temperature below the LC isotropization temperature, differential scanning calorimetry (DSC) indicated that well-defined structural changes occurred with increasing temperature. In the previous study, therefore, the optical microscopic observations indicating the isotropization process for the polymer, were combined with DSC and viscoelastic property results to identify the LC range of this polymer. 
In the present report we present the use of another optical method, polarized light transmission, which was found to be a very sensitive method for following the transition processes of this type of LCP. Also presented are physical property data obtained on a lower molecular weight sample, Polymer II, of the same polymer which was recently prepared in our laboratories.

\section{EXPERIMENTAL}

Polymer I, which had an inherent viscosity of $0.99 \mathrm{dlg}^{-1}$ in tetrachloromethane at 0.5 $\mathrm{g} \mathrm{dl}^{-1}$ and $40^{\circ} \mathrm{C}$, was prepared by a melt polymerization reaction. In an effort to obtain a lower molecular weight polymer, Polymer II was prepared by solution polymerization. Under identical conditions the inherent viscosity of this polymer was $0.17 \mathrm{dlg}^{-1}$. IR spectroscopy showed that the two polymers were structurally indistinguishable. In the previous study it was found that Polymer I had a weight average molecular weight of 80,000 , compared to polystyrene standards, by gel permeation chromatograph (GPC), and a suprisingly broad molecular distribution (MWD) of 4.1.

An identical procedure was used to obtain sample pieces from Polymer II as from Polymer I for measuring viscoelastic properties by a Rheometrics Mechanical Spectrometer (RMS), ${ }^{1}$ but when Polymer II was dried under vacuum for $5 \mathrm{~h}$ at approximately $100^{\circ} \mathrm{C}$ it visibly fused inside the vacuum oven after several hours at that temperature. Molding Polymer II at $185^{\circ} \mathrm{C}$ produced transparent samples that were brittle and difficult to remove from the mold, but samples molded at $135^{\circ} \mathrm{C}$ were much less transparent and more easily removable.

Because only a limited amount of Polymer II was available, samples were molded only for $20 \mathrm{~mm}$ parallel plate measurements. The actual sample thickness for viscoelastic property measurement was about $0.85 \mathrm{~mm}$. A strain amplitude of $5 \%$ was employed. At $140^{\circ} \mathrm{C}$ no effect of strain amplitude, $\gamma_{0}$, was seen between $1 \%$ and $10 \%$ at an angular frequency $\omega=$ $10 \mathrm{~s}^{-1}$.

The polarized light transmission method was performed on both polymers by the procedure described recently, ${ }^{2}$ which involves the measurement of total transmitted light intensity under parallel polarizers, that is, no analyzer was used in the light path above the sample. A similar method in which light transmission was measured with crossed polarizers has been described for other LCPs. ${ }^{3}$

\section{RESULTS AND DISCUSSION}

The results of the dynamic viscoelastic temperature sweep experiment for Polymer II are shown in Figure 1. There, measurements reveal the presence of only slight intermediate temperature shoulders for the lower molecular weight polymer in contrast to distinct intermediate temperature minima observed in the dynamic viscoelastic properties for Polymer I. ${ }^{1}$ For the storage modulus $G^{\prime}$, the shoulder is in the range of $95-105^{\circ} \mathrm{C}$, while that for the loss modulus $G^{\prime \prime}$ occurs in the range of $100-110^{\circ} \mathrm{C}$. The absolute value of the complex viscosity $\left|\eta^{*}\right|$ also shows a shoulder in this temperature

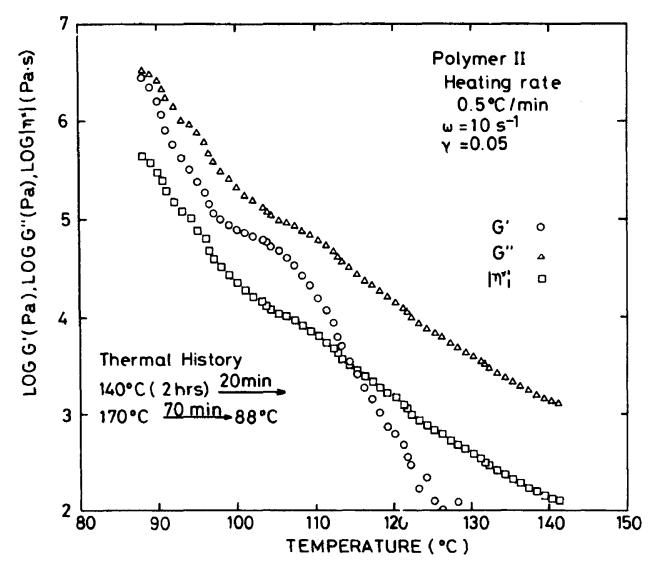

Figure 1. Dynamic viscoelastic properties for Polymer II on heating at approximately $0.5^{\circ} \mathrm{C} \mathrm{min}^{-1}$; measurements made at $\omega=10 \mathrm{~s}^{-1}$ and $\gamma_{0}=5 \%$. 
range. The lower molecular weight of Polymer II results in a lowering of the $T_{\mathrm{m}}$ (melting) and $T_{\mathrm{i}}$ (isotropization) transitions, and a decrease in $\Delta T$, namely, the difference between $T_{\mathrm{i}}$ and $T_{\mathrm{m}} \cdot{ }^{4}$ The narrowing of $\Delta T$ may cause an overlapping of effects, masking the expected minimum of viscoelastic functions in an intermediate temperature range. Although not included here, nearly identical curves were seen for the RMS measurements on cooling the sample from high temperatures, as was shown previously for Polymer I. ${ }^{1}$

Polarized light transmission studies with parallel polarizers were carried out on both polymers, each of which was either: [A] quenched from the isotropic state or [B] slow cooled from the isotropic state before the measurements. The results for Polymer I are shown in Figure 2. For the quenched sample, (a) in Figure 2, the polarized light transmission values were initially high, corresponding to that expected for a sample which has been quenched into the isotropic state. On heating the quenched sample, a sharp decrease in light transmission occurred close to the $T_{\mathrm{g}}$ obtained for Polymer $I$ in the earlier DSC measurements. ${ }^{1}$ As the temperature of the sample increased the transmitted intensity, in arbitrary units, changed rather abruptly from a strongly negative slope, to a zero slope in the range of the $T_{\mathrm{m}}$ transition previously measured by DSC, to a strongly positive slope. In contrast the slow-cooled sample, (b), had an initially very low light transmission value, as expected for a sample with a structured morphology capable of scattering most of the incident light.

There is a need with LCPs for more sensitive methods of characterizing the isotropization process than either optical microscopy or DSC. The latter is particularly insensitive for LC copolyesters, which often show little or no indication of an endothermic behavior that can be associated with the isotropization process. The viscoelastic properties maximum for the temperature sweep, as described in the previous report, is somewhat effective as an indication

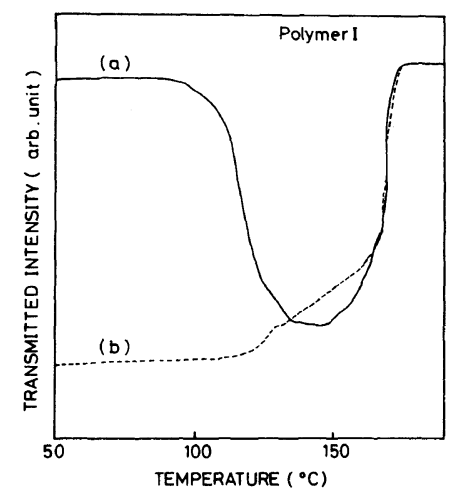

Figure 2. Light transmission using parallel polarizers during the heating cycle of Polymer I $\left(10^{\circ} \mathrm{C} \mathrm{min}^{-1}\right)$ : (a) sample quenched from the isotropic state; (b) sample slow-cooled from the isotropic state.

of this process, but not in an absolute sense. That is, the RMS temperature sweep method is quite sentitive to the occurrence of transitions, but it gives no indication of the type of transition involved.

In another study, light transmission with crossed polars was interpreted as giving an earlier indication than polarized light microscopy of the beginning of isotropization, ${ }^{3}$ and it discussed the course of separation in relation to isotropization for TLCPs copolyesters. The controlling factors identified were molecular weight distribution and copolymer composition. For copolyesters with weak isotropization transitions, this transition could be characterized quite well with the use of cross polarized light transmission, but not by DSC. ${ }^{3}$

Phase separation of LC copolyesters has also been investigated in recent reports using polarized light microscopy, ${ }^{5}$ and by dielectric methods. ${ }^{6,7}$ In the polarized light investigation referred to above, ${ }^{3}$ a homopolymer and two copolymers clearly showed the initial stages of phase separation well before isotropization. The phase separation isotropization for the homopolymer had a particularly narrow temperature range.

In the present study, the isotropization temperature of Polymer I was first char- 


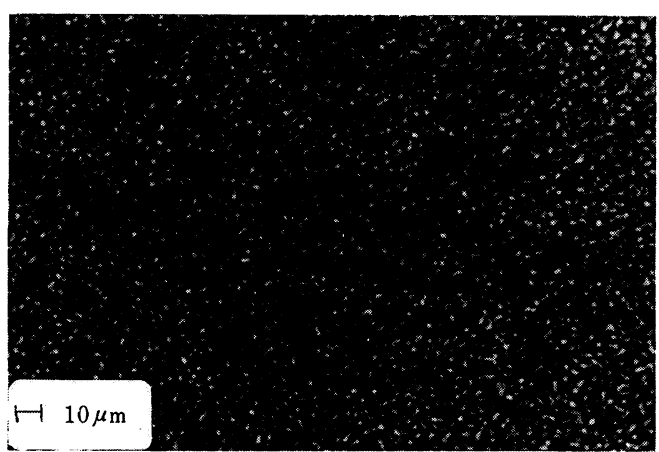

(a)

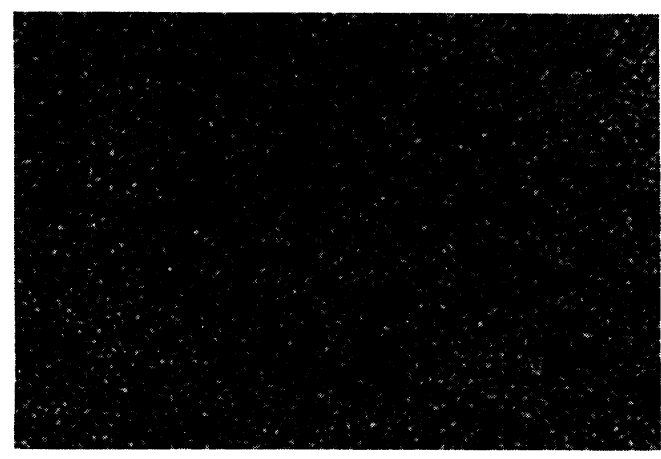

(b)

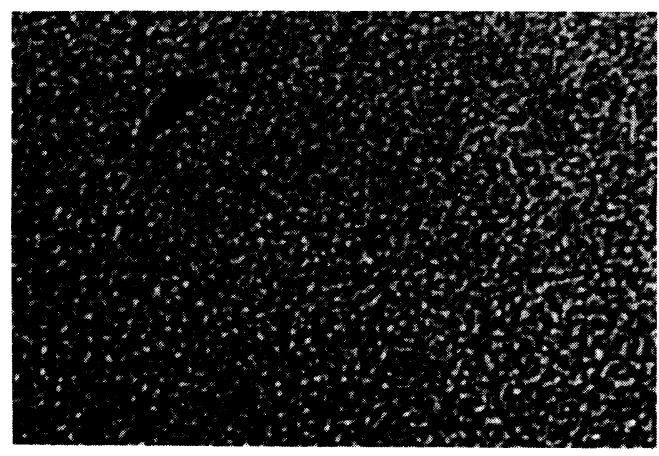

(c)

Figure 3: Photomicrographs of the phase separation process of Polymer I: (a) at $172.7^{\circ} \mathrm{C}$ after heating slowly in the liquid crystalline state; (b) at $172.7^{\circ} \mathrm{C}$ after $18 \mathrm{~min}$, at the same location as (a); and (c) at $172.7^{\circ} \mathrm{C}$ after $36 \mathrm{~min}$, at a different location from (a) and (b).

acterized in detail by polarized light microscopy with the results shown in Figure 3, which contains a series of photomicrographs taken at

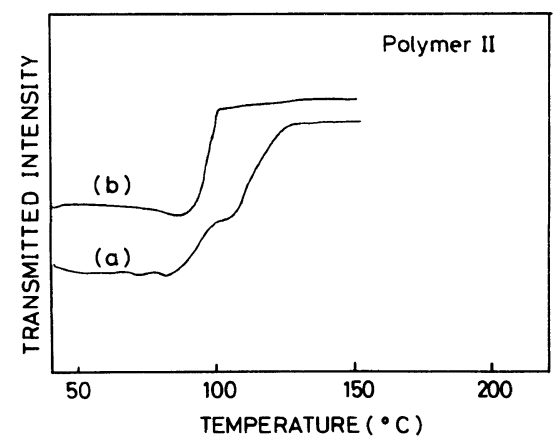

Figure 4. Light transmission with parallel polarizers for the heating cycle of Polymer II $\left(10^{\circ} \mathrm{C} \mathrm{min}{ }^{-1}\right)$ : (a) results for the powder sample; (b) results for the annealed film.

$172.7^{\circ} \mathrm{C}$ over a period of $36 \mathrm{~min}$. This series of photomicrographs shows, in a semi-qualitative manner, the course of isotropization which is revealed in a much more quantitative manner by the transmission data polotted in Figure 2(a). Similar results, in which light transmission was found to increase linearly throughout the isotropization process, were reported for a LC copolyester, and for a blend of the LCP with a non-LCP in the initial report of the use of this technique by Bhattacharya and Lenz. ${ }^{2}$ The light transmission results also correspond very closely with the results obtained from dynamic viscoeleastic property temperature sweep study for Polymer I. ${ }^{1}$ Both methods appear to be useful for quantitatively characterizing isotropization and the accompanying phase separation, which could not be followed in this manner for Polymer I, by either DSC or polarized light microscopy.

For Polymer II, two types of samples were evaluated for application of the light transmission method with the results shown in Figure 4. One sample, which was obtained directly from the polymerization reaction after precipitation, was heated as a powder, (a), and the other was heated in the form of an annealed film, (b). The results from both measurements show a similar increase in transmitted light intensity close to $90^{\circ} \mathrm{C}$. For the film, (b), there was a sharp increase in intensity up to $100^{\circ} \mathrm{C}$, 


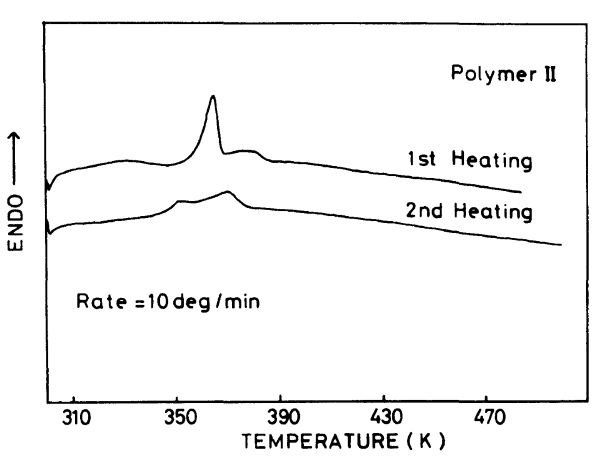

Figure 5. First and second DSC heating cycle $\left(10^{\circ} \mathrm{C}\right.$ $\min ^{-1}$ ) for Polymer II.

while for the powder sample, (a), a second step increase is seen in the vicinity of $100^{\circ} \mathrm{C}$, with the transmitted light intensity finally reaching its maximum value at $127^{\circ} \mathrm{C}$. From DSC analysis, an endothermic peak in the region of $110^{\circ} \mathrm{C}$, which is present in the initial heating, disappeared on subsequent heating after the sample had initially been heated to elevated temperature, as shown in Figure 5. Indeed, the previous study showed that the thermal transitions were better defined for Polymer I in the second DSC heating cycle. ${ }^{1}$

The results for the DSC, RMS, and light transmission studies on Polymer II, after it had been initially heated to elevated temperature, were all consistent. For the film, the light transmission results, in the region of the rapid increase and the subsequent attainment of a maximum value of light transmission with increasing temperature, defined the range in which the step change was seen in RMS viscoelastic property results. The second heating cycle in the DSC showed a recurrent maximum in the range of $90-95^{\circ} \mathrm{C}$, with an occasional peak present in the range of $70^{\circ} \mathrm{C}$. Because of the small value of $\Delta T$, the higher temperature peak may be a combination of the $T_{\mathrm{m}}$ and $T_{\mathrm{i}}$ peaks. The lower temperature endothermic peak which is sometimes present in the vicinity of $70^{\circ} \mathrm{C}$ was previously observed to occur between $T_{\mathrm{m}}$ and $T_{\mathrm{i}}$ for this and other thermotropic LCPs. ${ }^{3}$

The high temperature endothermic peak at about $110^{\circ} \mathrm{C}$ in the initial DSC heating cycle, and the increase at about $110^{\circ} \mathrm{C}$ on the approach to the maximum value obtained at $127^{\circ} \mathrm{C}$ observed in the light transmission study, which was not observed in the second heating, may be attributed to the lack of well defined transitions for Polymer II because of its low molecular weight, which could result in a substantial overlap of the $T_{\mathrm{g}}, T_{\mathrm{m}}$, and $T_{\mathrm{i}}$ transitions.

Acknowledgements. We would like to express our appreciation to the Ministry of Education, Science, and Culture of Japan (Monbusho) for Grant-in-Aid for Scientific Research (Nos. 61470101 and 63850181) to TM, to Monbusho, which financially supported the research stay in Japan of PD, and to the Materials Research Laboratory of the University of Massachusetts, which is funded by the National Science Foundation, for the support of SB.

\section{REFERENCES}

1. P. Driscoll, K. Fujiwara, T. Masuda, A. Furukawa, and R. W. Lenz, Polym. J., 20, 351 (1988).

2. S. K. Bthattacharya and R. W. Lenz, J. Polym. Sci., Polym. Phys. Ed., in press.

3. S. L. Wunder, S. Ramachandran, C. R. Gochanour, and M. Weinburg, Macromolecules, 19, 1696(1986).

4. R. W. Lenż, Faraday Dis., 79, 21 (1985).

5. T. Shiwaku, A. Nakai, H. Hasegawa, and T. Hashimoto, Polym. Commun., 28, 174 (1987).

6. R. S. Benson and D. N. Lewis, Polym. Commun., 28, 289 (1987).

7. U. W. Gedde, D. Buerger, and R. H. Boyd, Macromolecules, 20, 988 (1987). 